University of Wollongong

Research Online

Faculty of Engineering - Papers (Archive)

Faculty of Engineering and Information

Sciences

$1-1-2012$

\title{
Ultrafast dynamics of the Mn3+ d-d transition and spin-lattice interaction in YMnO3 film
}

Z Jin

Shanghai University

Hong Ma

Shanghai University

Gaofang Li

Shanghai University

Y Xu

Shanghai University

$\mathrm{G} \mathrm{Ma}$

Shanghai University

See next page for additional authors

Follow this and additional works at: https://ro.uow.edu.au/engpapers

Part of the Engineering Commons

https://ro.uow.edu.au/engpapers/5035

\section{Recommended Citation}

Jin, Z; Ma, Hong; Li, Gaofang; Xu, Y; Ma, G; and Cheng, Zhenxiang: Ultrafast dynamics of the Mn3+ d-d transition and spin-lattice interaction in YMnO3 film 2012.

https://ro.uow.edu.au/engpapers/5035

Research Online is the open access institutional repository for the University of Wollongong. For further information contact the UOW Library: research-pubs@uow.edu.au 


\section{Authors}

Z Jin, Hong Ma, Gaofang Li, Y Xu, G Ma, and Zhenxiang Cheng 


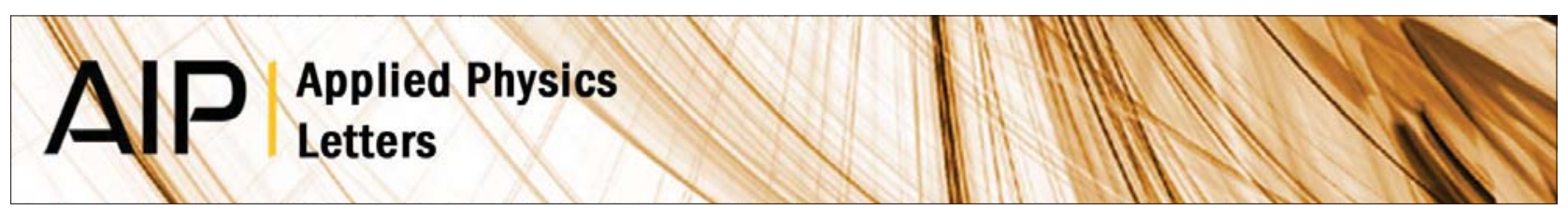

\section{Ultrafast dynamics of the Mn3+ d-d transition and spin-lattice interaction in YMnO3 film}

Zuanming Jin, Hong Ma, Gaofang Li, Yue Xu, Guohong Ma et al.

Citation: Appl. Phys. Lett. 100, 021106 (2012); doi: 10.1063/1.3675906

View online: http://dx.doi.org/10.1063/1.3675906

View Table of Contents: http://apl.aip.org/resource/1/APPLAB/v100/i2

Published by the American Institute of Physics.

\section{Related Articles}

First-principles study of charging effect on magnetism of Pd (100) ultrathin films J. Appl. Phys. 112, 073910 (2012)

Magnetization of $2.6 \mathrm{~T}$ in gadolinium thin films

Appl. Phys. Lett. 101, 142407 (2012)

Bit error rate investigation of spin-transfer-switched magnetic tunnel junctions Appl. Phys. Lett. 101, 142406 (2012)

Perpendicular magnetic anisotropy of cobalt films intercalated under graphene Appl. Phys. Lett. 101, 142403 (2012)

Electric field assisted sputtering of $\mathrm{Fe} 3 \mathrm{O} 4$ thin films and reduction in anti-phase boundaries J. Appl. Phys. 112, 073909 (2012)

\section{Additional information on Appl. Phys. Lett.}

Journal Homepage: http://apl.aip.org/

Journal Information: http://apl.aip.org/about/about_the_journal

Top downloads: http://apl.aip.org/features/most_downloaded

Information for Authors: http://apl.aip.org/authors

\section{ADVERTISEMENT}

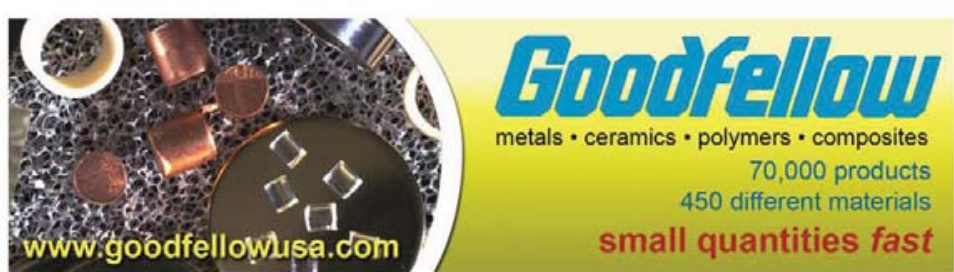




\title{
Ultrafast dynamics of the $\mathrm{Mn}^{3+} \mathrm{d}$-d transition and spin-lattice interaction in $\mathrm{YMnO}_{3}$ film
}

\author{
Zuanming Jin, ${ }^{1}$ Hong Ma, ${ }^{1}$ Gaofang Li, ${ }^{1}$ Yue Xu, ${ }^{1}$ Guohong Ma, ${ }^{1, a)}$ and Zhenxiang Cheng ${ }^{2}$ \\ ${ }^{1}$ Department of Physics, Shanghai University, 99 Shangda Road, Shanghai 200444, \\ People's Republic of China \\ ${ }^{2}$ Institute for Superconductor and Electronic Materials, University of Wollongong, Squair Ways, \\ North Wollongong, New South Wales 2500, Australia
}

(Received 13 October 2011; accepted 19 December 2011; published online 9 January 2012)

\begin{abstract}
We investigate the photo-induced carrier dynamics and spin-lattice interaction in hexagonal $\mathrm{YMnO}_{3}$ film by the temperature-dependent femtosecond pump-probe spectroscopy. The spin-lattice interaction is identified from the slow component of the transient transmittance change with the excitation energies tuned to $1.7 \mathrm{eV}$ and $2.0 \mathrm{eV}$, which are close to $\mathrm{Mn}^{3+}$ ions $d_{(x z),(y z)} \rightarrow d_{\left(z^{2}\right)}$ and $d_{\left(x^{2}-y^{2}\right),(x y)} \rightarrow d_{\left(z^{2}\right)}$ transition, respectively. Temperature dependences of the spin-lattice relaxation parameters demonstrate that the spin-lattice interaction is strongly connected with the d-d transition within $\mathrm{Mn}^{3+}$ ions and enhanced by spin ordering. (C) 2012 American Institute of Physics. [doi:10.1063/1.3675906]
\end{abstract}

In recent years, multiferroic oxides have attracted intense attention because of the great potential for application in the fields of oxide electronics and spintronics. ${ }^{1-3}$ The physical properties of multiferroicity are extremely complicated due to the intrinsic integration and strong coupling between ferroelectricity and magnetism. ${ }^{4,5}$ The microscopic origin of the magnetoelectric phenomenon has been interpreted as the magnetoelastic effect, ${ }^{6}$ which eventually leads to an effective coupling between electric dipole moment and magnetic moment. Therefore, the understanding of the strong coupling among the charge, spin, orbital, and lattice degrees of freedom is a central topic of multiferroic oxide materials. Conventional studies of spin-lattice coupling in multiferroic oxides were investigated by steady-state spectroscopy, such as inelastic neutron scattering, ${ }^{7}$ Raman spectra, ${ }^{8}$ and thermal expansion measurements, ${ }^{9}$ etc. In the last two decades, ultrafast optical spectroscopy has been demonstrated to provide valuable insights into the microscopic dynamics in materials as reported in Refs. 10-16. In particular, the temperature-dependent pump-probe spectroscopy has proven to be an effective tool to study the electron-phonon, phonon-phonon, and spin-phonon interactions. ${ }^{12-16}$

Ogasawara et al. showed that the complex correlation between charge, lattice, and spin in various ferromagnetic and ferrimagnetic compounds can be explicitly identified from the different characteristic time scales by time-resolved magneto-optical Kerr spectroscopy (TRMOKE). ${ }^{10}$ Wall et al. studied the ultrafast coupling between coherent lattice vibrations and the magnetic structure of a strong spin-orbit coupling structure, $\mathrm{LaMnO}_{3} .{ }^{11} \mathrm{~A}$ magnetic resonance had been observed in multiferroic $\mathrm{Ba}_{0.6} \mathrm{Sr}_{1.4} \mathrm{Zn}_{2} \mathrm{Fe}_{12} \mathrm{O}_{22}$ using pump-probe reflectance spectroscopy, which revealed that the dielectric tensor of material can be modulated by magnetization precession owing to the magnetoelectric effect. ${ }^{12}$ The intrinsic interdependence between the optical conductivity and magnetism in colossal magneto-resistance manganites $\mathrm{La}_{0.7} \mathrm{D}_{0.3} \mathrm{MnO}_{3}(\mathrm{D}=\mathrm{Ca}, \mathrm{Sr})$ was investigated by Lobad

${ }^{a)}$ Electronic mail: ghma@staff.shu.edu.cn. et al., which allowed for the determination of the spin-lattice coupling magnitude. ${ }^{13,14}$ Shih et al. investigated the ultrafast photoinduced electron and phonon dynamics of $\mathrm{HoMnO}_{3}$ single crystal, and the anomalous thermoelastic effect around $\mathrm{T}_{\mathrm{N}}$ was reported. ${ }^{15,16}$ These studies demonstrate that the ultrafast optical spectroscopy, even in non-Kerr experimental configurations, is an alternative method to explore the interaction of spin, charge, and lattice degrees of freedom in magnetic oxides. ${ }^{17}$

$\mathrm{YMnO}_{3}$ in its hexagonal phase is one of the few multiferroic materials where ferroelectric and antiferromagnetic ordering coexist. ${ }^{18}$ In contrast to the other rare-earth ions in magnetic oxides, such as $\mathrm{Ho}^{3+}\left(4 \mathrm{f}^{10}\right), \mathrm{Er}^{3+}\left(4 \mathrm{f}^{11}\right), \mathrm{Tm}^{3+}\left(4 \mathrm{f}^{12}\right)$, and $\mathrm{Yb}^{3+}\left(4 \mathrm{f}^{13}\right)$, the magnetic properties of $\mathrm{YMnO}_{3}$ solely originate from $\mathrm{Mn}^{3+}$ ions. Thus, $\mathrm{YMnO}_{3}$ provides a pure material system for the study of interaction among charge, spin, and lattice. Hexagonal $\mathrm{YMnO}_{3}$ is formed by stacked $\mathrm{Mn}-\mathrm{O}$ and $\mathrm{Y}-\mathrm{O}$ layers, in which the $\mathrm{Mn}^{3+}$ ions are surrounded by three in-plane and two apical oxygen ions $\left(\mathrm{MnO}_{5}\right.$ bipyramide $)$ and form a triangular lattice. The Mn spins order antiferromagnetically below $\mathrm{T}_{\mathrm{N}} \sim 80 \mathrm{~K}$, and the spins lie in the hexagonal plane, orthogonal to the Mn-O bonds. Using optical pump-probe spectroscopy, Jang et al. reported a coherent $31 \mathrm{GHz}$ acoustic phonon above the magnetic ordering temperature and a $\mathrm{THz}$ frequency coherent mode in the antiferromagnetic phase in $\mathrm{YMnO}_{3}$ single crystals, which demonstrated that the acoustic phonon is coupled to spin ordering. ${ }^{19}$ Kimel et al. found that the nonlinearity in $\mathrm{YMnO}_{3}$ results in a transient perturbation of the dielectric permittivity tensor. ${ }^{20} \mathrm{Up}$ to now, the investigation of the carrier dynamics and spin-lattice interaction in $\mathrm{YMnO}_{3}$ film is lacking compared with $\mathrm{HoMnO}_{3}$ and $\mathrm{LuMnO}_{3}$. In this letter, spin-lattice interaction in hexagonal $\mathrm{YMnO}_{3}$ film was investigated by femtosecond pumpprobe spectroscopy with varying temperature, which spans the antiferromagnetic transition temperature. Temperature dependences of the spin-lattice interaction parameters at two excitation energies reveal that the spin-lattice interaction is strongly connected with both the d-d transition within $\mathrm{Mn}^{3+}$ ions and the spin ordering. 

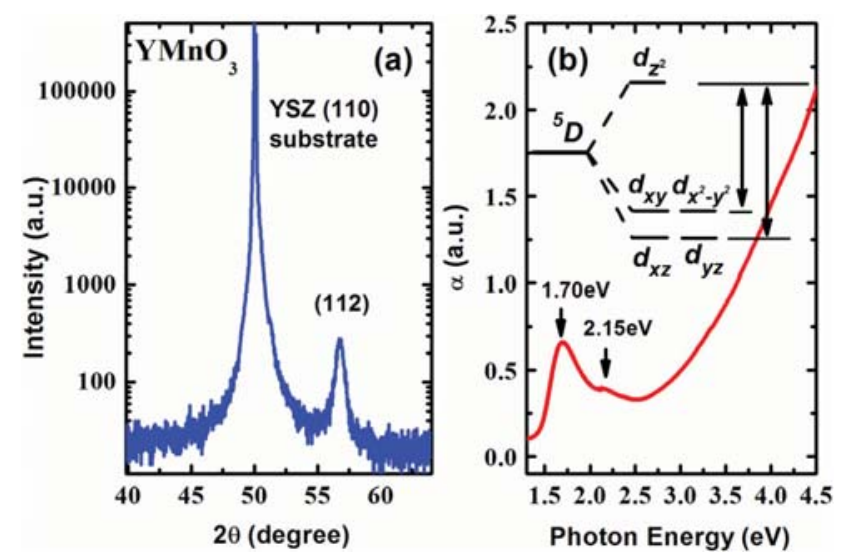

FIG. 1. (Color online) (a) XRD patterns and (b) the UV-Vis absorption spectrum for hexagonal $\mathrm{YMnO}_{3}$ film. The inset illustrates the $3 \mathrm{~d}$ orbit splitting of the $\mathrm{Mn}^{3+}$ ion in trigonal bipyramid environment.

The $\mathrm{YMnO}_{3}$ film on YSZ (110) substrate was fabricated by a pulsed laser deposition system with a $355 \mathrm{~nm}$-laser source at a repetition rate of $10 \mathrm{~Hz}$. $\mathrm{YMnO}_{3}$ targets were synthesized by a standard solid-state reaction method from the starting chemicals $\mathrm{Y}_{2} \mathrm{O}_{3}$ and $\mathrm{MnCO}_{3}$, with purity of $99.99 \%$. The deposition was carried out at $800{ }^{\circ} \mathrm{C}$ with a dynamic oxygen pressure of 20 mtorr. The x-ray diffraction (XRD) pattern of the $\mathrm{YMnO}_{3}$ film shown in Fig. 1(a) was determined with a JEOL $3500 \mathrm{x}$-ray diffractometer, which shows the $\mathrm{YMnO}_{3}$ film is epitaxially grown on YSZ (110) with an orientation of $\langle 112\rangle$. The inset of Fig. 1(b) shows the electronic energy diagram as derived from the local symmetry of $\mathrm{Mn}^{3+}$ ion. ${ }^{21}$ Four $3 \mathrm{~d}$ electrons of the $\mathrm{Mn}^{3+}$ occupy the ground state ${ }^{5} \mathrm{D}$, a quintet state. In hexagonal $\mathrm{YMnO}_{3}$, the ${ }^{5} \mathrm{D}$ state splits into three states under the influence of a moderate crystalline field resulting from the trigonal bipyramidal symmetry $\overline{6} \mathrm{~m} 2$. Fig. 1(b) shows the room temperature absorption spectra for the hexagonal $\mathrm{YMnO}_{3}$ film. Two absorption bands appear, a strong band around $1.70 \mathrm{eV}$ and a weak one around $2.15 \mathrm{eV}$, which are attributed to inter-site optical transitions from the hybridized occupied states with $d_{\left(x^{2}-y^{2}\right),(x y)}$ and $d_{(x z),(y z)}$ orbital symmetry to the unoccupied $\mathrm{Mn} d_{\left(z^{2}\right)}$ state, respectively. Charge-transfer transitions between $\mathrm{O} 2 \mathrm{p}$ and $\mathrm{Mn} 3 \mathrm{~d}$ are expected to occur above $3 \mathrm{eV}$.

In time-resolved optical experiments, a pump beam excites a sample initiating a dynamical response that is monitored by a time delayed probe beam. The source of excitation consists of a mode-locked Ti:Sapphire oscillator (Mai Tai HP-1020) generating $100 \mathrm{fs}$ pulses at $800 \mathrm{~nm}$. A regenerative amplifier system (Spectra-Physics, Spitfire Pro) was used to amplify the pulses $10^{6}$ times at a repetition rate of $1 \mathrm{kHz}$. Part of the energy was used to pump an optical parametric amplifier (Topas-C) for generating different wavelengths ultrashort pulses. Pump and probe beam were focused by a $50 \mathrm{~cm}$ focal length lens and overlapped on the same spot of the sample with a spot size of $500 \mu \mathrm{m}$. The ratio between the power of pump and probe beams was 50:1. The typical fluence of the pump pulses was set at $\sim 0.8 \mathrm{~mJ} / \mathrm{cm}^{2}$. The sample was mounted in a closed-cycle liquid-He cryostat in vacuum chamber with four optically accessible windows. A mechanical delay line was used to vary the arrival time of pump pulses related to probe pulses. In order to improve the immunity of laser drift, the pump beam was chopped at $\sim 490 \mathrm{~Hz}$, and the probe signal was detected by a silicon photodiode connected to a lock-in amplifier.

Figures 2(a) and 2(b) show the typical temperaturedependent transient transmittance change $(\Delta \mathrm{T} / \mathrm{T})$ for the hexagonal $\mathrm{YMnO}_{3}$ film obtained at two excitation energies, $1.70 \mathrm{eV}$ and $2.0 \mathrm{eV}$, respectively. The initial rising (or falling) components of the $\Delta \mathrm{T} / \mathrm{T}$ indicate the $\mathrm{Mn}^{3+} \mathrm{d}-\mathrm{d}$ transition in $\mathrm{YMnO}_{3}$, which means the carriers transit from ground state to excited state induced by the pump pulses. The amplitudes of the initial components start to diminish around $\mathrm{T}=100 \mathrm{~K}$, which is due to the $\mathrm{Mn}^{3+} \mathrm{d}$-d transition blocken when d-d transition energy level undergoes a blueshift with decreasing temperature. ${ }^{16,22}$ Following the initial parts, $\Delta \mathrm{T} / \mathrm{T}$ curves contain three primary relaxation components: (1) the ultrafast component ( $<1 \mathrm{ps})$ corresponds to the electron-lattice thermalization, (2) the subsequent slow component gives a direct information of the energy exchange between the lattice and spin system, and (3) after these two relaxation processes, a quasi-constant component corresponds to either the remagnetization process or the heat diffusion out of the illuminated area of the sample. These three processes $(t>0)$ can be described by the following equation phenomenologically:

$$
\Delta T / T=A \exp \left(-t / \tau_{\text {fast }}\right)+B \exp \left(-t / \tau_{\text {slow }}\right)+T_{\infty},
$$

where A and B are the amplitudes of the fast and slow components mentioned above, respectively. $\tau_{\text {fast }}$ and $\tau_{\text {slow }}$ are the relaxation time constant of the fast and slow components, respectively. $T_{\infty}$ is a time-independent quasi-constant parameter, which should recover to the ground state after a much longer time delay (hundreds of picoseconds-several nanoseconds). The experimental data are well fitted using the Eq. (1), which is shown in Figs. 2(a) and 2(b) as the black solid lines. Hereinafter, we focus on the discussion of
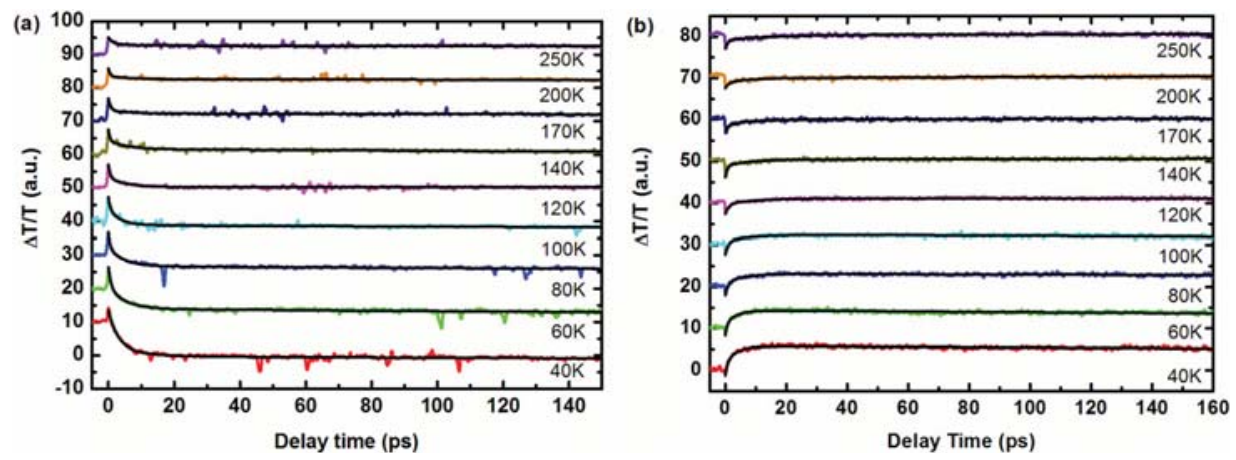

FIG. 2. (Color online) Relaxation curves of the relative transmittance change $\Delta \mathrm{T} / \mathrm{T}$ of $\mathrm{YMnO}_{3}$ film at two excitation energies: (a) $1.7 \mathrm{eV}$ and (b) $2.0 \mathrm{eV}$. The temperatures marked in the figure are the steady-state temperatures of sample. $\Delta \mathrm{T} / \mathrm{T}$ curves are shifted along the vertical axis for clarity. The black solid lines show the fitting results by Eq. (1). 
temperature dependence of the slow component parameters, which is considered to relate to the spin-lattice interaction mediated by the spin-orbit coupling. The fitting fast component parameters, $\tau_{\text {fast }}$ and $\mathrm{A}$, contain the information about the electron-phonon scattering, which will be discussed in another publication. $^{22}$

The main results (which are derived from the fitting data of Fig. 2) are presented in Fig. 3. Figure 3(a) shows the temperature dependences of $\tau_{\text {slow }}$ with excitation energies at $1.70 \mathrm{eV}$ and $2.0 \mathrm{eV}$, respectively. The spin-lattice interaction time displays the nonmonotonic temperature dependences, and the peaks of $\tau_{\text {slow }}$ appear at $\sim 120 \mathrm{~K}$ with values of $\sim 12$ ps $(2.0 \mathrm{eV})$ and $\sim 6 \mathrm{ps}(1.70 \mathrm{eV})$. The observed nonmonotonic temperature dependences are indeed anticipated. Excited electrons are assumed to shed their excess energy after thermalized within the first picoseconds. A threetemperature model can be invoked to model the weak coupling between lattice and spin freedom of degrees, which is coupled by anisotropy fluctuations of the crystal field. ${ }^{23,24}$ The spin-lattice interaction time is related to the lattice specific heat $C_{l}$, spin specific heat $C_{s}$, and spin-lattice energy coupling constant $G_{s l}$ as $\tau_{s-l} \approx \frac{C_{s} C_{l}}{G_{s l}\left(C_{s}+C_{l}\right)} \cdot{ }^{13,24,25}$ Under a mean field approximation, the temperature dependent $C_{s}(T)$ is proportional to $\partial M^{2} / \partial T$, where $M$ is the sublattice magnetization as a function of temperarute. ${ }^{26}$ For weak lattice heating, the magnetic specific heat $C_{S}(T)$ contributes to the peak of the temperature-dependent spin-lattice relaxation time, ${ }^{25}$ which was also observed in $\mathrm{La}_{0.7} \mathrm{Ca}_{0.3} \mathrm{MnO}_{3}$ and $\mathrm{La}_{0.7} \mathrm{Sr}_{0.3} \mathrm{MnO}_{3} .{ }^{13}$ We note here, however, the critical temperature $\left(\mathrm{T}_{\mathrm{c}} \sim 120 \mathrm{~K}\right)$ is higher than the Néel temperature $\left(\mathrm{T}_{\mathrm{N}} \sim 80 \mathrm{~K}\right)$ of $\mathrm{YMnO}_{3}$ crystal, which might be caused by the short-range spin ordering. It has been reported that the short-range correlations fluctuate both in space and time above $\mathrm{T}_{\mathrm{N}}$ in $\mathrm{YMnO}_{3}$. This short-range spin ordering is a signature of a spin liquid phase, which forms out of geometrically frustrated Mn moments. ${ }^{27}$ It is further found that, at the temperature window from 70 to $200 \mathrm{~K}$, the $\tau_{\text {slow }}$ at excitation energy of $1.70 \mathrm{eV}$ is faster than that at $2.0 \mathrm{eV}$, which reveals that the spin-lattice interaction is sensitive to the photoexcitation energy. In other words, when the excitation energy
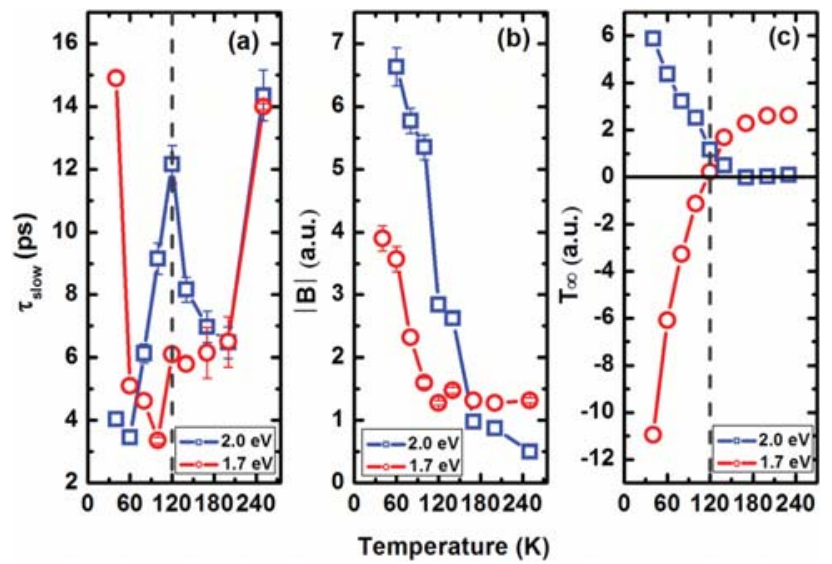

FIG. 3. (Color online) The temperature dependences of fitting parameters given by Eq. (1): (a) time constants $\tau_{\text {slow }}$, (b) amplitudes of slow component $\mathrm{B}$, and (c) background signal $T_{\infty}$ for two excited energies, $1.7 \mathrm{eV}$ and $2.0 \mathrm{eV}$. Vertical broken lines correspond to $\mathrm{T}_{\mathrm{c}}=120 \mathrm{~K}$. is approaching to $d_{\left(x^{2}-y^{2}\right),(x y)} \rightarrow d_{\left(z^{2}\right)}$ transition, the d-electrons of $\left(x^{2}-y^{2}, x y\right)$ state could be excited, and then it will significantly affect the ordering of the $\mathrm{Mn}^{3+}$ spins. ${ }^{28,29}$ From our data, the spin-lattice coupling constant, $G_{s l}$, is estimated to be $\sim 75 \times 10^{10}$ and $\sim 37 \times 10^{10} \mathrm{~W} /($ Kmole $)$ for the excitation energies of $1.7 \mathrm{eV}$ and $2.0 \mathrm{eV}$, respectively. $G_{s l}$ was calculated by taking the lattice specific heat $\mathrm{C}_{1} \sim 44 \mathrm{~J} /(\mathrm{K}$ mole) and the spin specific heat $\mathrm{C}_{\mathrm{s}} \sim 5 \mathrm{~J} /(\mathrm{K}$ mole $) .{ }^{30}$ In addition, the lower the temperature is, in our case, lower than $70 \mathrm{~K}$ for $2.0 \mathrm{eV}$ and $100 \mathrm{~K}$ for $1.7 \mathrm{eV}$, the more robust the magnetic ordering becomes due to the prevailing of the longrange antiferromagnetic ordering, as a result, a longer time is needed to disturb the spin system, which is in accordance with our observation. Similar phenomenon was also reported in $\mathrm{HoMnO}_{3}$ single crystals. ${ }^{16}$

Figure 3(b) shows the temperature dependence of slowcomponent-amplitude, B, collected at the excitation energies of $1.70 \mathrm{eV}$ and $2.0 \mathrm{eV}$, respectively. From our experimental results, it is expected that the intensity of the spin-lattice interaction increases markedly with decreasing temperature. We attribute this behavior to the competition between shortand long-range antiferromagnetism in $\mathrm{YMnO}_{3}$, which causes the significant spin-scattering behavior and then enhances the spin-lattice interaction. ${ }^{19}$

As is clearly seen in the Fig. 3(c), for excitation energy at $1.70 \mathrm{eV}$, the quasi-constant parameter $T_{\infty}$ gradually decreases with decreasing temperature and alters its sign from positive to negative when temperature is approaching $\sim 120 \mathrm{~K}$. Nevertheless, for the excitation energy at $2.0 \mathrm{eV}, T_{\infty}$ increases with decreasing temperature and tends to be zero above $120 \mathrm{~K}$. This temperature dependence of $T_{\infty}$ is relevant to the magnetism-induced redshift in the optical conductivity spectra at lower temperature. ${ }^{3,14,16,31}$ Ren et al. ascribed this longlived component to a slow spin-relaxation process of the magnetically ordered phase in $\mathrm{La}_{0.67} \mathrm{Ca}_{0.33} \mathrm{MnO}_{3}{ }^{32}$ Shih et al . reported that the temperature dependence of the amplitude of the negative component is similar to the displacement of $\mathrm{Mn}$ atoms, which is directly related with the magnetic ordering of the $\mathrm{Mn}$ moments in $\mathrm{HoMnO}_{3}{ }^{15}$

In summary, the ultrafast dynamics probed by temperature-tunable femtosecond pump-probe spectroscopy at two excitation energies are performed to investigate the spinlattice interaction in hexagonal $\mathrm{YMnO}_{3}$ film. Our data show that the fast component in $\Delta \mathrm{T} / \mathrm{T}$ corresponds to the electronlattice thermalization, and the subsequent slow component analyzed by a three-temperature model represents the energy exchange among the electron, spin, and lattice system. Temperature dependences of slow component parameters indicate that the spin-lattice interaction in hexagonal $\mathrm{YMnO}_{3}$ film is strongly connected with the d-d transition within $\mathrm{Mn}^{3+}$ ions and significantly enhanced by spin ordering.

The research is supported by National Natural Science Foundation of China (11174195) and Science and Technology Commission of Shanghai municipal (09530501100). Z. $\mathrm{X}$. Cheng thanks Australia Research Council for support through a Future Fellowship.

${ }^{1}$ Y. Tokura, Phys. Today 56(7), 50 (2003).

${ }^{2}$ J. Ma, J. Hu, Z. Li, and C.-W. Nan, Adv. Mater. 23, 1062 (2011). 
${ }^{3}$ D. N. Basov, R. D. Averitt, D. van der Marel, M. Dressel, and K. Haule, Rev. Mod. Phys. 83, 471 (2011).

${ }^{4}$ C. Zhong, Q. Jiang, H. Zhang, and X. Jiang, Appl. Phys. Lett. 94, 224107 (2009).

${ }^{5}$ K.-J. Jang, J. Lim, J. Ahn, J.-H. Kim, K.-J. Yee, J. S. Ahn, and S.-W. Cheong, New J. Phys. 12, 023017 (2010).

${ }^{6}$ S. Lee, A. Pirogov, M. Kang, K.-H. Jang, M. Yonemura, T. Kamiyama, S.-W. Cheong, F. Gozzo, N. Shin, H. Kimura, Y. Noda, and J.-G. Park, Nature 451, 805 (2008).

${ }^{7}$ S. Petit, F. Moussa, M. Hennion, S. Pailhès, L. Pinsard-Gaudart, and A. Ivanov, Phys. Rev. Lett. 99, 266604 (2007).

${ }^{8}$ J. Vermette, S. Jandl, A. A. Mukhin, V. Y. Ivanov, A. Balbashov, M. M. Gospodinov, and L. Pinsard-Gaudart, J. Phys.: Condens. Matter 22, 356002 (2010).

${ }^{9}$ C. dela Cruz, F. Yen, B. Lorenz, Y. Q. Wang, Y. Y. Sun, M. M. Gospodinov, and C. W. Chu, Phys. Rev. B 71, 060407(R) (2005).

${ }^{10}$ T. Ogasawara, K. Ohgushi, Y. Tomioka, K. S. Takahashi, H. Okamoto, M. Kawasaki, and Y. Tokura, Phys. Rev. Lett. 94, 087202 (2005).

${ }^{11}$ S. Wall, D. Prabhakaran, A. T. Boothroyd, and A. Cavalleri, Phys. Rev. Lett. 103, 097402 (2009).

${ }^{12}$ D. Talbayev, S. A. Trugman, A. V. Balatsky, T. Kimura, A. J. Taylor, and R. D. Averitt, Phys. Rev. Lett. 101, 097603 (2008).

${ }^{13}$ A. I. Lobad, R. D. Averitt, C. Kwon, and A. J. Taylor, Appl. Phys. Lett. 77, 4025 (2000)

${ }^{14}$ A. I. Lobad, A. J. Taylor, C. Kwon, S. A. Trugman, and T. R. Gosnell, Chem. Phys. 251, 227 (2000).

${ }^{15}$ H. C. Shih, L. Y. Chen, C. W. Luo, K. H. Wu, J.-Y. Lin, J. Y. Juang, T. M. Uen, J. M. Lee, J. M. Chen, and T. Kobayashi, New J. Phys. 13, 053003 (2011).

${ }^{16}$ H. C. Shih, T. H. Lin, C. W. Luo, J.-Y. Lin, T. M. Uen, J. Y. Juang, K. H Wu, J. M. Lee, J. M. Chen, and T. Kobayashi, Phys. Rev. B 80, 024427 (2009).
${ }^{17}$ J. S. Dodge, A. B. Schumacher, J.-Y. Bigot, D. S. Chemla, N. Ingle, and M. R. Beasley, Phys. Rev. Lett. 83, 4650 (1999)

${ }^{18}$ H. L. Yakel, W. C. Koehler, E. F. Bertaut, and E. Forrat, Acta Crystallogr. 16, 957 (1963).

${ }^{19}$ K.-J. Jang, H.-G. Lee, S. Lee, J. Ahn, J. S. Ahn, N. Hur, and S.-W. Cheong, Appl. Phys. Lett. 97, 031914 (2010).

${ }^{20}$ A. V. Kimel, R. V. Pisarev, F. Bentivegna, and Th. Rasing, Phys. Rev. B 64, 201103(R) (2001)

${ }^{21}$ C. Degenhardt, M. Fiebig, D. Fröhlich, Th. Lottermoser, and R. V. Pisarev, Appl. Phys. B 73, 139 (2001).

${ }^{22}$ Y. Xu, Z. M. Jin, G. H. Ma, and Z. X. Cheng, "Ultrafast Spectroscopy of the $\mathrm{Mn}^{3+}$ d-d Transition on $\mathrm{YMnO}_{3}$ Film" (unpublished).

${ }^{23}$ W. Hübner and K. H. Bennemann, Phys. Rev. B 53, 3422 (1996).

${ }^{24}$ G. M. Müller, J. Walowski, M. Djordjevic, G.-X. Miao, A. Gupta, A. V. Ramos, K. Gehrke, V. Moshnyaga, K. Samwer, J. Schmalhorst, A. Thomas, A. Hütten, G. Reiss, J. S. Moodera, and M. Münzenberg, Nature Mater. 8, 56 (2009).

${ }^{25}$ Q. Zhang, A. V. Nurmikko, G. X. Miao, G. Xiao, and A. Gupta, Phys. Rev. B 74, 064414 (2006)

${ }^{26}$ D. H. Martin, Magnetism in Solids (MIT, Cambridge, MA, 1967).

${ }^{27}$ J. Park, J.-G. Park, G. S. Jeon, H.-Y. Choi, C. Lee, W. Jo, R. Bewley, K. A. McEwen, and T. G. Perring, Phys. Rev. B 68, 104426 (2003).

${ }^{28}$ T. Katsufuji, S. Mori, M. Masaki, Y. Moritomo, N. Yamamoto, and H. Takagi, Phys. Rev. B 64, 104419 (2001).

${ }^{29}$ K. Murakami, T. Yamauchi, A. Nakamura, Y. Moritomo, H. Tanaka, and T. Kawai, Phys. Rev. B 73, 180403(R) (2006).

${ }^{30}$ D. G. Tomuta, S. Ramakrishnan, G. J. Nieuwenhuys, and J. A. Mydosh, J. Phys.: Condens. Matter 13, 4543 (2001).

${ }^{31}$ R. C. Rai, J. Cao, J. L. Musfeldt, S. B. Kim, S.-W. Cheong, and X. Wei, Phys. Rev. B 75, 184414 (2007).

${ }^{32}$ Y. H. Ren, M. Ebrahim, H. B. Zhao, G. Lüpke, Z. A. Xu, V. Adyam, and Q. Li, Phys. Rev. B 78, 014408 (2008). 